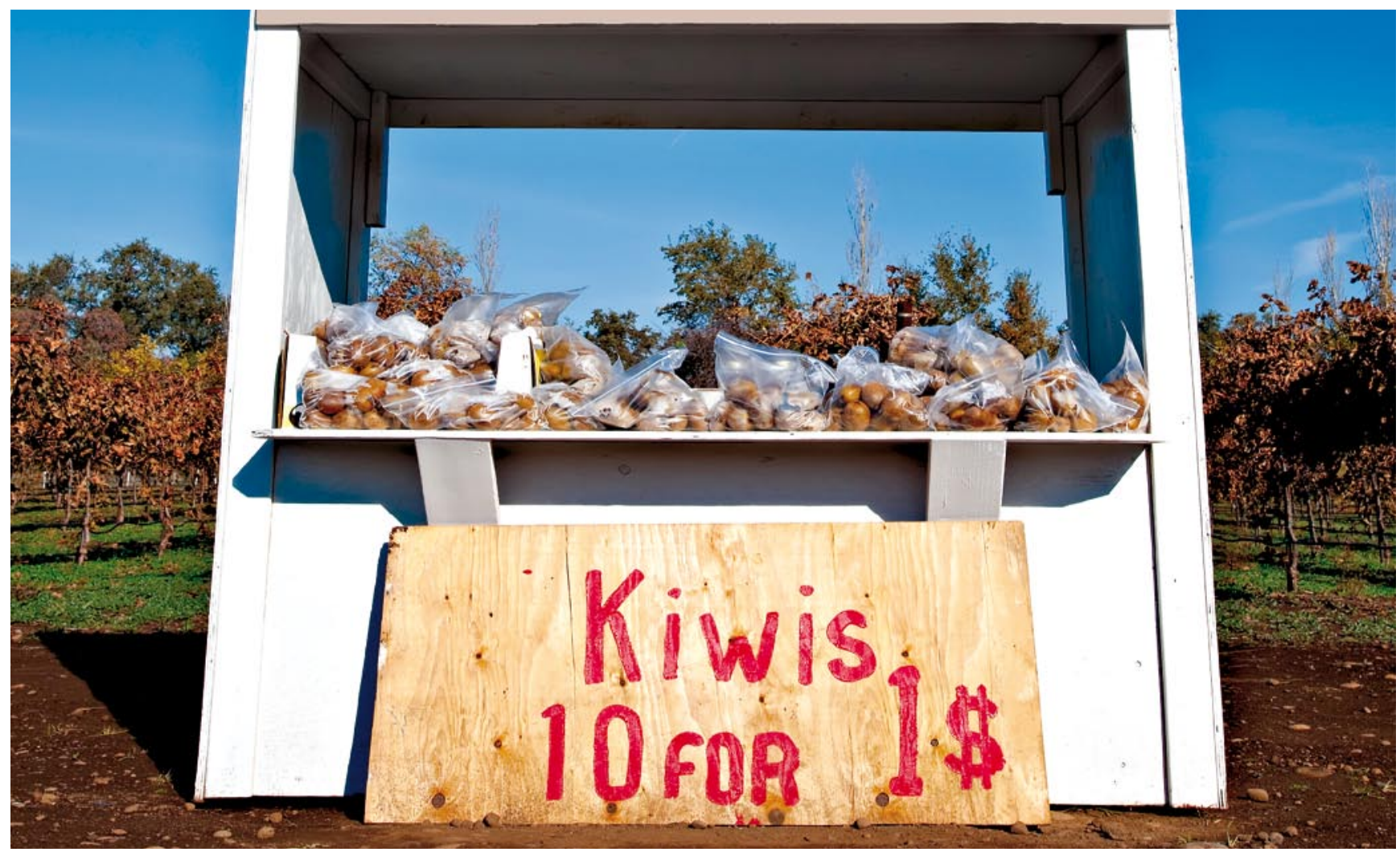

\title{
Neue Wege zum Kunden - Innovationen in der Kunde-Bank-Interaktion
}

\section{Die Interaktion einer Bank mit ihren Kunden hat sich in den vergangenen Jahrzehnten deutlich} weiterentwickelt. Zwar sind auch nach dem E-Business-Hype die physischen Filialnetze weiterhin von Bedeutung, jedoch hat die Entwicklung zu stärkerer Beratung und dem zunehmenden Einsatz von Informationstechnologie (IT) sowohl die Filialen selbst als auch die Interaktionskanäle insgesamt verändert. Der vorliegende Beitrag beschreibt die Ergebnisse einer strukturierten Beispielsammlung innovativen IT-Einsatzes im Bereich der Kunde-Bank-Interaktion.

Von Prof. Dr. Rainer Alt, Till Möwes und Dr. Thomas Puschmann

$\mathrm{D}$ er klassische Bankschalter, an dem der Kunde mit einem hinter Sicherheitsglas agierenden Bankangestellten standardisierte Transaktionen wie etwa Ein- und Auszahlungen oder Überweisungen erledigt hat, ist einem beratungsorientierten Ansatz gewichen. Während mit Automaten und Electronic Banking IT-basierte Innovationen das Massengeschäft übernahmen, zeigen die offenen Filialkonzepte den zunehmenden Beratungsschwerpunkt der Bankangestellten. Beispiele wie „Shop-in-Shop“Ansätze in der Zukunftsfiliale Q110 bei der Deutschen Bank oder das auf eine Verbindung von betreuter Selbstbedienung abzielende „Einzonenmodell“ der Münchner Bank beruhen nicht zu- letzt auf einem zunehmenden IT-Einsatz bei den Beratungsaktivitäten. Zusätzlich entstehen mit der Virtualisierung der Bankaktivitäten über das Internet und der Verbreitung intuitiver mobiler Technologien zunehmend Möglichkeiten zur Beratung ohne (physischen) Kundenberater. Wie bereits in der Vergangenheit können auch diese technologischen Innovationen deutliche Veränderungen in den etablierten Branchenstrukturen und Geschäftsprozessen bedeuten.

Grundsätzlich verbindet die Bank-Kunde-Interaktion die in den Kundenprozessen von Retail-, Privat- und Firmenkunden enthaltenen Bedürfnisse über mehrere Interaktionskanäle mit 
Tab. 1 Innovationen in der Kunde-Bank-Interaktion nach Bankprozessen

\begin{tabular}{|c|c|c|}
\hline Prozessbereich & Kanal & Beispiel \\
\hline \multirow{3}{*}{$\begin{array}{l}\text { Beratung } \\
\text { und Vertrieb }\end{array}$} & Filiale & $\begin{array}{l}\text { Beratungsgespräch mit interaktiver Bildoberfläche (z. B. Multitouch-Beratungstisch bei Q110), teilautomatische } \\
\text { Formularerfassung und -verarbeitung (z. B. Digipen) }\end{array}$ \\
\hline & Online & $\begin{array}{l}\text { Interaktive Videokommunikation (z. B. WebDesk bei Nykredit Bank), Finanznachrichten-Abonnements, E-Loyalty } \\
\text { (z. B. studienkredite.de), virtuelle Bankfilialen (z. B. Second Life), Produktkonfigurator (z. B. DZ Bank), Single-Sign- } \\
\text { On-Portale (z. B. Filiale 5.0 der Sparkassen) }\end{array}$ \\
\hline & Mobile & Mobile Marketing (z. B. Raiffeisenbank Information Services), Finanznachrichten-Abonnements \\
\hline \multirow{3}{*}{$\begin{array}{l}\text { Zahlungs- } \\
\text { verkehr }\end{array}$} & $\begin{array}{l}\text { Handel/Point } \\
\text { of Sale (POS) }\end{array}$ & Zahlungsinstitut (z. B. Montrada) \\
\hline & Online & $\begin{array}{l}\text { Virtuelle Kreditkarte (z. B. Wirecard), Online Zahlungssysteme (z. B. PayPal), alternative Währungen (z. B. Linden } \\
\text { Dollars, Facebook), elektronische Rechnungsabwicklung (z. B. VR-Rechnungsservice, SIX Paynet, PostFinance } \\
\text { E-Rechnung), SMS-Sicherheitsverfahren (z. B. Credit Suisse) }\end{array}$ \\
\hline & Mobile & $\begin{array}{l}\text { Multibankfähige Zahlungsverkehrsapplikationen (z. B. iOutBank), kontaktlose Zahlungen mittels Nearfield } \\
\text { Communication Technologie (z. B. Kooperationen Apple/Visa, Nokia/O2 Télefonica), SMS-Sicherheitsverfahren, } \\
\text { intelligente Geldbörsen (z. B. Proverbial Wallet) }\end{array}$ \\
\hline \multirow{3}{*}{$\begin{array}{l}\text { Anlage und } \\
\text { Finanzieren }\end{array}$} & Filiale & Multimediaplattformen mit Videos und Nachrichten (z. B. Bloomberg, Thomson Reuters) \\
\hline & Online & $\begin{array}{l}\text { Community Banking (z. B. Fidor Bank), Bankless Banking (z. B. Smava), Kreditmanagement (z. B. KM3 der S\&N), } \\
\text { Finanzverwaltung (z. B. Kontoblick, Thrive, iPlanner) }\end{array}$ \\
\hline & Mobile & Mobile Finanzplanung (z. B. iPlanner, Budget), Mobile Brokerage (z. B. Cortal Consors) \\
\hline \multirow{3}{*}{ Übergreifend } & Filiale & $\begin{array}{l}\text { Financial-Planning- und Risk-Profiling-Applikationen zum Abgleich von Kundenbedürfnissen und Anlagestrategien } \\
\text { in der Beratung (z. B. FinaMetrica, struktur AG etc.) }\end{array}$ \\
\hline & Online & Social Banking (z. B. Noa Bank), Elektronische ID (z. B. BankID), Vergleichsplattformen (z. B. ciao.de, openforum.de) \\
\hline & Mobile & Smartphone Apps (z. B. Postbank iBanking, VR Banken Filialfinder App) \\
\hline
\end{tabular}

den Frontoffice-Bereichen einer Bank. Zu Letzteren zählen neben Beratung und Vertrieb vor allem der Zahlungsverkehr sowie der Anlage- und Finanzierungsbereich. Diese Leistungen nutzen Bankkunden nach einer jüngeren Befragung immer stärker elektronisch und mobil: So gingen 67 Prozent der Befragten von einer stärkeren Nutzung elektronischer Kanäle bis 2015 aus, während dies für den Kanal Filiale gerade einmal fünf Prozent tun [1]. Dies unterlegen die Nutzerzahlen von E-Banking in Deutschland, die in einem Jahr um zwei Millionen Nutzer auf nunmehr 26 Millionen angestiegen sind (BITKOM 2010) und eine aktuelle Befragung, worin Privatkunden eine IT-gestützte Beratung befürworten [2]. Welche weiteren innovativen Perspektiven existieren nun über bekannte Ansätze wie das E-Banking hinaus? Dazu hat das Kompetenzzentrum „Sourcing in der Finanzindustrie“ der Universitäten St. Gallen und Leipzig (www. ccsourcing.org) über 50 Ansätze analysiert, welche die KundeBank Interaktion zukünftig beeinflussen können. Wie Tabelle 1 zeigt, finden sich Beispiele für beinahe sämtliche Interaktionskanäle, Bankprozesse und Kundengruppen. Für jeden Prozessbereich sind nachfolgend jeweils zwei Innovationen exemplarisch beschrieben.

\section{Innovationen in Beratung und Vertrieb}

Einen Beitrag zur Verbesserung der filialbasierten Beratung bilden interaktive Bildoberflächen auf Touchscreens unterschiedlicher Größe. Dabei navigiert der Berater gemeinsam mit dem Kunden durch Anwendungen und hat die Möglichkeit, komplexe Zusammenhänge durch Visualisierungen und Simulationen zu erklären. So könnte etwa ein strukturiertes Anlageprodukt mit Hebelwirkung, Basiswerten und Rückzahlungswerten dargestellt sein, um dadurch dem Kunden die komplexen Funktionszusammenhänge zu verdeutlichen. Weiterhin erkennen Geräte, wie etwa das in der Filiale Q110 der Deutschen Bank zur Finanzberatung eingesetzte Surface von Microsoft, aufgelegte Objekte und verknüpfen diese 


\section{STRATEGIE}

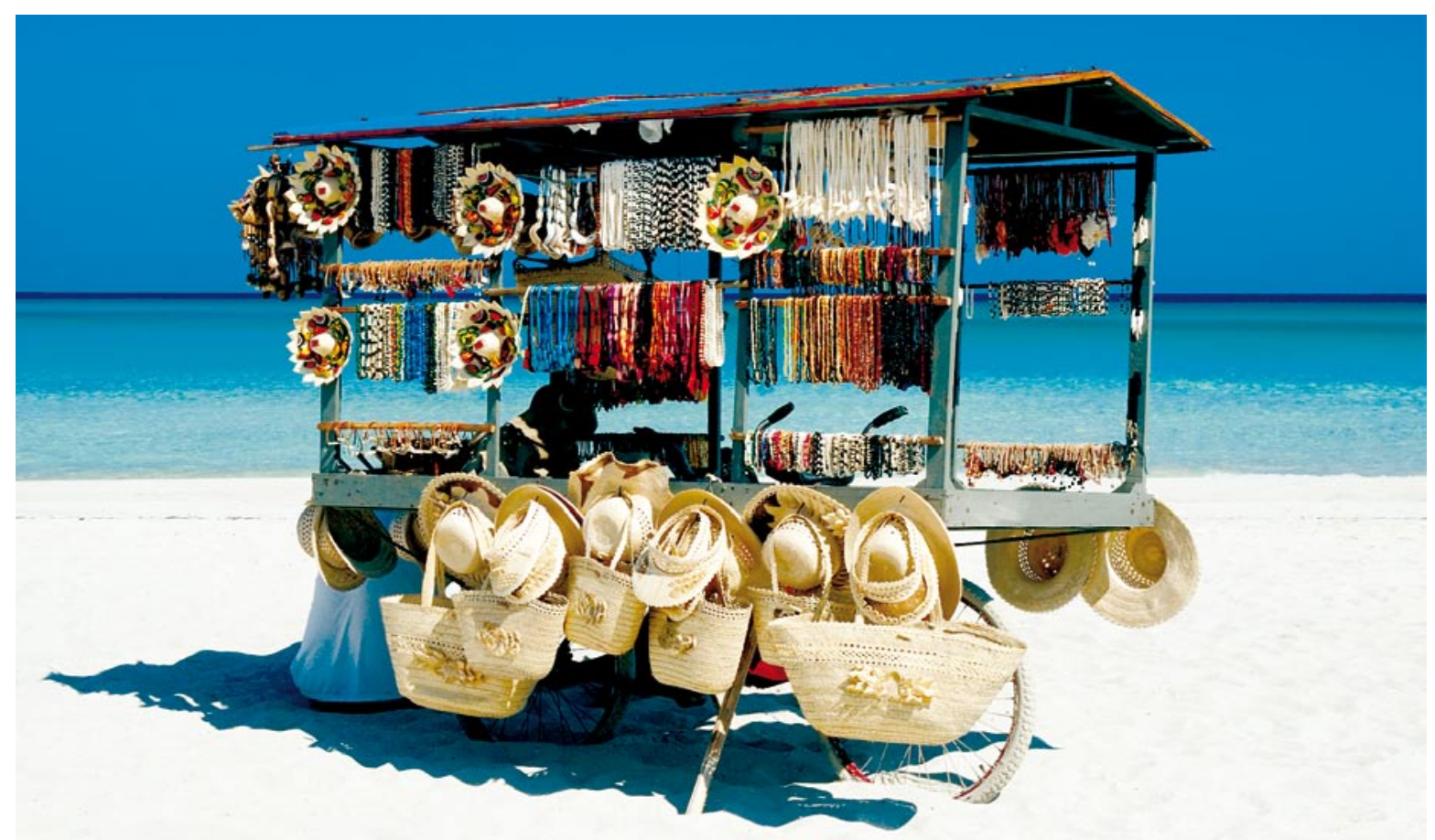

anschließend mit Informationen, beispielweise einen physischen Prospekt zu einem Fonds mit dessen jüngsten Wertentwicklungen. Das Mitwirken der Kunden auf dem Beratungstisch bindet diese stärker in den Beratungsprozess ein und verringert die Distanz zwischen Berater und Kunden, was auch zu einer stärkeren emotionalen Bindung der Kunden führen soll. Eine weitere Entwicklung in dem Bereich sind Tablet-PCs der neueren Generation in Verbindung mit Touch-Technologie. Gegenüber stationären Beratungstischen erlauben touch-fähige Notebooks wie das iPad eine günstigere und mobilere Form der interaktiven Beratung. Allerdings verändern die innovativen Multitouch-Lösungen den eigentlichen Beratungsprozess bislang noch wenig, was nahelegt, sie mit den unten beschriebenen grundlegenderen Ansätzen der interaktiven Wertschöpfung wie Mass Customization oder der Open Innovation zu verbinden [3].

Ein weiteres Innovationsbeispiel ist der Einsatz von Videokonferenzsystemen. Diese zielen auf die Überbrückung räumlicher Distanzen bei der Beratung, wie es beispielsweise bei global arbeitenden Kunden mit lokal stationierten Beratern im Private Banking sinnvoll ist. Weil Videokonferenzen mit ihrem hohen Anteil analoger Kommunikationselemente wie Bild und Sprache der persönlichen Interaktion nahekommen, haben diese insbesondere im Wissenstransfer bei komplexen Fragestellungen Verwendung gefunden. So nutzen Pharmaunternehmen seit mehreren Jahren unter der Bezeichnung „Video-Detailing“ die webbasierte interaktive Produktberatung. Ein erstes Beispiel für den Einsatz im Retail Banking ist WebDesk, die interaktive Videokommunikationsplattform der dänischen Nykredit Bank. Diese ermöglicht neben der Kommunikation über Video, Audio und Chat den sicheren Dokumentenaustausch sowie grafische Simulationen zur Erklärung der von internetbasierten Beratungstools generierten numerischen Ergebnisse.

\section{Innovationen im Zahlungsverkehr}

Im bereits mit E-Banking und Electronic Bill Presentment \& Payment (EBPP) im Frontend sowie Lösungen wie SWIFT im Backend hochautomatisierten Zahlungsverkehr treten Zahlungsinstitute und virtuelle Kreditkarten als Innovationen hervor. Zahlungsinstitute sind Folge einer bis zum 1. November 2009 in den Mitgliedsstaaten der EG umzusetzenden Zahlungsdiensterichtlinie im Rahmen des einheitlichen Euro-Zahlungsraums (SEPA). Diese soll unter anderem gewährleisten, dass neue Zahlungsdienstleister Zugang zum Markt erhalten und führt daher Zah-

\section{"Virtuelle Kreditkarten beinhalten in der Regel keinen Kreditrahmen und sind genau genommen Guthaben- beziehungsweise Prepaid-Karten. «}

lungsinstitute als neue Kategorie von Zahlungsdienstleistern ein. Dabei handelt es sich um reine Zahlungsdienstleister, die keine Einlagen entgegennehmen und kein elektronisches Geld ausgeben. Da die Zulassung als Zahlungsinstitut in allen EU-Mitgliedsstaaten gilt, können Anbieter wie etwa montrada ihre Autorisierungs- und Abrechnungsleistungen für Kredit, Debit- und Kundenkarten auch grenzüberschreitend anbieten. Händler und Retail-Kunden benötigen keine Verbindung zu einer Bank.

Im Unterschied zu einer klassischen Kreditkarte erhält der Nutzer bei der virtuellen Kreditkarte zwar die Daten einer Kreditkarte (Kartennummer, CVC2-Nummer, Ablaufdatum), nicht 

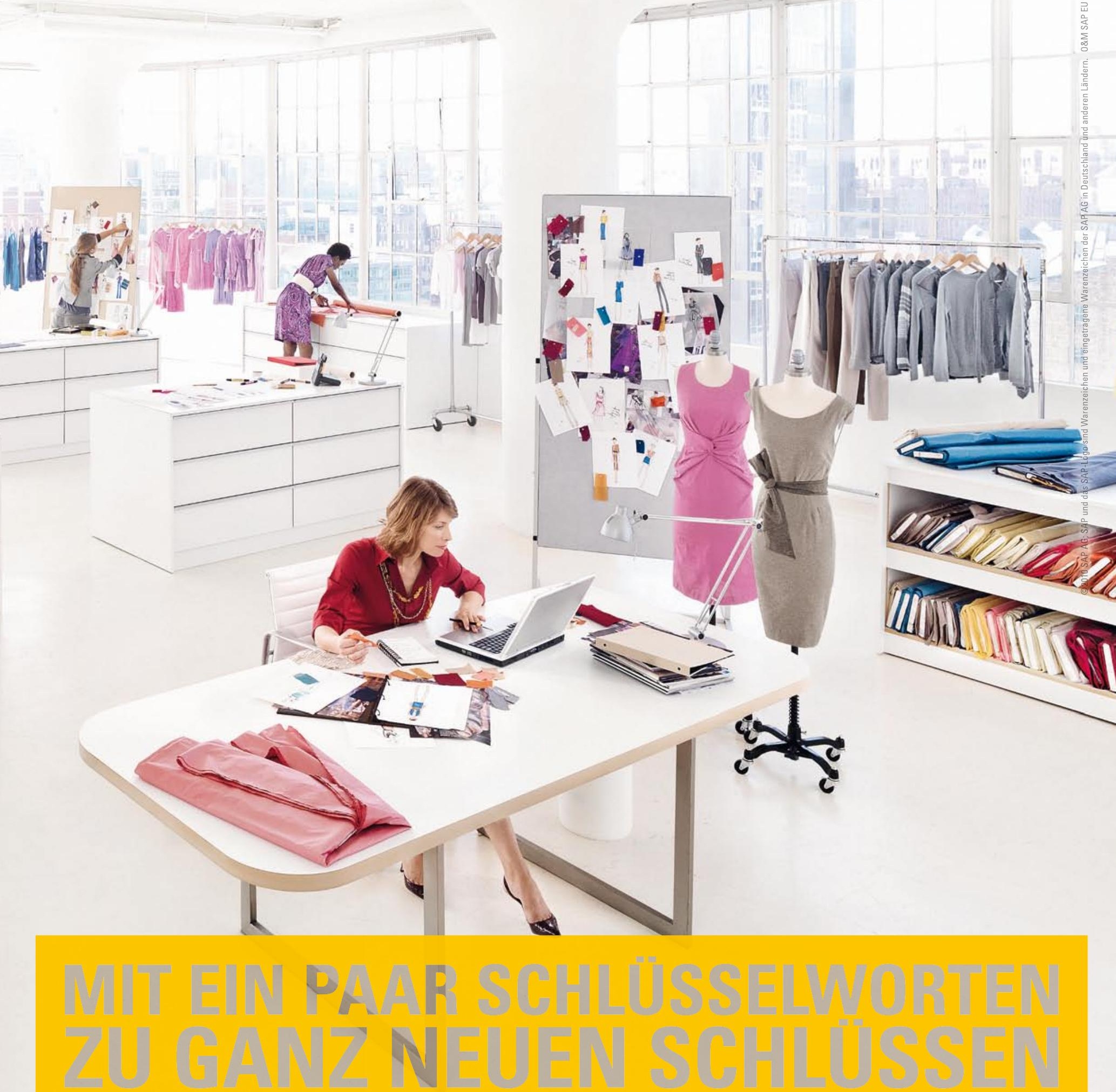

Stellen Sie sich vor, Sie könnten den Datendschungel Ihres Unternehmens ganz gezielt nach den Informationen durchforsten, die Sie im Augenblick für Ihre Entscheidungen brauchen. Sie selbst, nicht Ihr Datenbankspezialist. Schnell, einfach und intuitiv. Genau das macht SAP ${ }^{\circledR}$ Business0bjects ${ }^{\mathrm{TM}}$ Explorer möglich. Mit seiner Hilfe verdichten Sie gigantische Datenmengen in Sekundenschnelle zu aussagekräftigen Erkenntnissen: Welcher Hemdenschnitt ist nächstes Frühjahr angesagt? Wie kommt Wolle von den Färöern am günstigsten zu Ihnen? Mit SAP BusinessObjects Explorer finden Sie Antworten. Wie Sie mit seiner Hilfe mehr Klarheit in Ihr Unternehmen bringen, erfahren Sie unter sap.de/wissenfinden 
aber deren physisches Pendant. Virtuelle Kreditkartenangebote beinhalten in der Regel keinen Kreditrahmen und sind genau genommen Guthaben-(Prepaid-)Karten. So können Kunden beispielsweise mit der Wirecard in allen Onlineshops zahlen, die auch die MasterCard akzeptieren, und müssen für die Karte keine finanziellen Verpflichtungen wie Jahresgebühren eingehen. Gegenüber bestehenden Guthabenkarten haben sie aber bei Händlern die gleiche Akzeptanz wie klassische Kreditkarten. Im Vergleich zu diesen sind infolge des fehlenden Kreditrahmens für Händler die Betrugsmöglichkeiten geringer und für Kunden entfällt die Bonitätsprüfung.

\section{Innovationen in Anlage und Finanzierung}

In den Bereichen Anlage und Finanzierung finden sich das „Community Banking“ sowie der Ansatz der „Bankless Banks“ als neue Konzepte. Ersteres, auch „Mitmach-Banking“ genannt, greift die interaktive Wertschöpfung auf, die sich durch eine stärkere Einbindung des Kunden und größere Freiheitsgrade in der Leistungsgestaltung auszeichnet. So können beispielsweise Kunden der Fidor Bankähnlich dem „Wikipedia-Prinzip“ Finanzprodukte selbst erstellen, neue Produkte vorschlagen, sich untereinander beraten und erhalten für ihre Aktivitäten eine Vergütung. Die Beratung findet ausschließlich in der Online-Community statt, in der Kunden Finanzberater bewerten können. Lösungen des „CommunityBanking" setzen bekannte Web-2.0-Technologien wie Blogs, Chats und Diskussionsforen ein. Neu positionieren sich im Anlageumfeld auch Multimediaplattformen, die derzeit von Anbietern traditioneller Terminalsysteme für Finanznachrichten wie etwa Thomson Reuters oder Bloomberg aufgesetzt werden. Über diese Plattformen können zukünftig auch Endkunden mit aktuellen Informationen und Anlageempfehlungen versorgt werden.

Als zweiter Ansatz umfassen „Bankless Banks“ Online-Marktplätze zur Kreditvergabe zwischen Privatpersonen beziehungsweise zwischen Unternehmen. Smava stellt beispielsweise einen OnlineMarktplatz bereit, auf dem Kreditnehmer Anlageangebote machen und Kreditgeber die SCHUFA-geprüfte Bonität des potenziellen Kreditnehmers einsehen können. Bei Zustandekommen der Finanzierung gibt die im Hintergrund agierende Bank BIW dem Kreditnehmer einen Kredit, den sie an die eigentlichen Kreditgeber weiterveräußert. Das Modell ist für die Kreditparteien attraktiv, da die BIW Bank keine hohe Zinsmarge einfordert, sondern lediglich an Vermittlungsgebühren verdient. Außerdem betont es soziale Aspekte getreu dem Motto „Kredite von Mensch zu Mensch“, was für entsprechend motivierte Marktteilnehmer einen Anreiz zur Teilnahme darstellen kann. Die (Nicht-)Bank selbst verdient zwar nicht an der Zinsdifferenz, geht aber auch keine Kreditrisiken ein.

\section{Prozessbereichsübergreifende Innovationen}

Prozessbereichsübergreifend sind Innovationen, die mehrere Prozessbereiche (z. B. Beratung und Vertrieb, Zahlungsverkehr, Anlage) umfassen. Hierzu gehören beispielsweise neue Konzepte, die unter dem Stichwort „Financial Planning“ diskutiert werden und die ganzheitliche Beratung des Kunden umfassen, welche durch entsprechende Software unterstützt wird. Das Ziel besteht darin, für den Kunden ausgehend von seiner Einnahmen-/Ausgabensitutation und den vom Kunden definierten Zielen entsprechende Vorsorge-, Anlage- und Absicherungsstrategien zu ermitteln.

Weitere prozessbereichsübergreifende Innovationen umfassen mobile Technologien sowie Lösungen zur Informationssicherheit. Das bereits mehrfach mit wenig Erfolg von Banken initiierte „Mobile Banking“ hat durch intelligente Endgeräte mit intuitiver Multitouch-Benutzerführung wie das iPhone einen wichtigen

\section{» Mobile Banking hat durch intelli- gente Endgeräte mit intuitiver Benutzerführung einen wichtigen Wachstumsimpuls erhalten. "}

Wachstums- und Innovationsimpuls erhalten. $\mathrm{Zu}$ den seit Längerem bestehenden „Mobile Banking“-Plattformen kommen nun vielfältige Smartphone-Finanzapplikationen sowie Technologien des Web 2.0 wie Podcasts, RSS Feeds etc. hinzu. Auch Nicht-Banken drängen mit Angeboten auf den Markt, wie etwa PayPal mit einem handybasierten Zahlungsdienst. Die Postbank offeriert ihren Kunden neben der browserbasierten Lösung „iBanking“ auch eine „iPostbank“ genannte Applikation für das iPhone. Den Nutzern stehen die wichtigsten Onlinebanking-Funktionalitäten sowie ein Geldautomaten- und Filialfinder zur Verfügung. Der Postbank bietet das Mobile Banking zudem die Möglichkeit mobiler Marketingaktionen. Aus Kundensicht dürften jedoch multibankfähige Lösungen wie etwa iOutBank den Vorteil bieten, mit mehreren Banken elektronisch interagieren zu können.

Onlinebanking ist von Beginn an mit Sicherheitslösungen verbunden. Ein jüngstes Beispiel ist die elektronische ID, welche die Identifikation bei Transaktionen sicherer machen und zugleich erleichtern soll. In Norwegen erlaubt beispielsweise das BankIDSystem, sich mit einem elektronischen Identifikations- und Signatursystem im Internet auszuweisen und Dokumente digital $\mathrm{zu}$ unterschreiben. Ein vergleichbares System existiert mit BankID in Schweden. Unterschriften mit BankID sind in der EU rechtsverbindlich, wodurch sich unter anderem bei der strengen Identifizierung am Anfang von Bankbeziehungen ein hohes Rationalisierungspotenzial ergibt. Die BankID ist erhältlich als Chipkarte und als „Soft Certificate“ (Zugangsdaten auf einem Server gespeichert). Für Deutschland könnte der ab November 2010 ausgestellte elektronische Personalausweis mit vergleichbaren Funktionalitäten ähnliche Möglichkeiten bieten. 


\section{Zusammenfassung und Trends}

Zunächst zeigen die betrachteten Beispiele, dass sich in allen wichtigen Prozessbereichen einer Bank innovative IT-basierte Lösungen finden. Dem Lebenszyklusstadium dieser Innovationen dürfte es geschuldet sein, dass sie primär isoliert voneinander und noch nicht abgestimmt anzutreffen sind. Hierin liegt eine wichtige differenzierende Perspektive. Diese erkennt, dass die Kunde-Bank-Interaktion zunehmend hybrid ist und elektronische und mobile Interaktionsformen zwar an Bedeutung gewinnen, die klassischen Kanäle jedoch nicht substituieren. Insbesondere gehen Prognosen von einer größeren Überschneidung der Kanäle aus [4]. Im Vordergrund steht folglich eine Abstimmung der Interaktionskanäle, die beispielsweise den Kanalwechsel zur Fortsetzung einer Online-Anfrage in der Filiale und die Nutzung gleicher Informationsbestände in allen Kanälen vorsieht. Vergleichbare Flexibilitätsanforderungen ergeben sich auch infolge der Kundenprozessorientierung, da sich die drei klassischen Segmente Retail-Banking-, Private-Bankingund Firmenkunden zwar unter anderem hinsichtlich ihrer Leistungen und ihres Nutzungsverhaltens unterscheiden, dabei aber auf einer möglichst ähnlichen Applikationsunterstützung aufbauen.

Abbildung 2 sieht dazu einen "Service Bus“ vor, worüber Anbieter kundensegment-, prozess- und kanalübergreifend Dienstleistungen bereitstellen. Im Mittelpunkt stehen Business Services der Kunden-, Abwicklungs- und Interbankenprozesse, die die Geschäftslogik kapseln und sich zu fachlichen Clustern zusammenfassen lassen [5]. Application Services wie zum Beispiel Services in CRM-Systemen setzen Business Services technisch um und sind häufig als Webservices bestehender Applikationen realisiert. Eine möglichst auch anbieterübergreifend konzipierte modulare Architektur begründet sich auch in den Trends, welche sich aus den Innovationsbeispielen ableiten:

\section{Abb. 1 Strukturierung der Kunde-Bank-Interaktion}

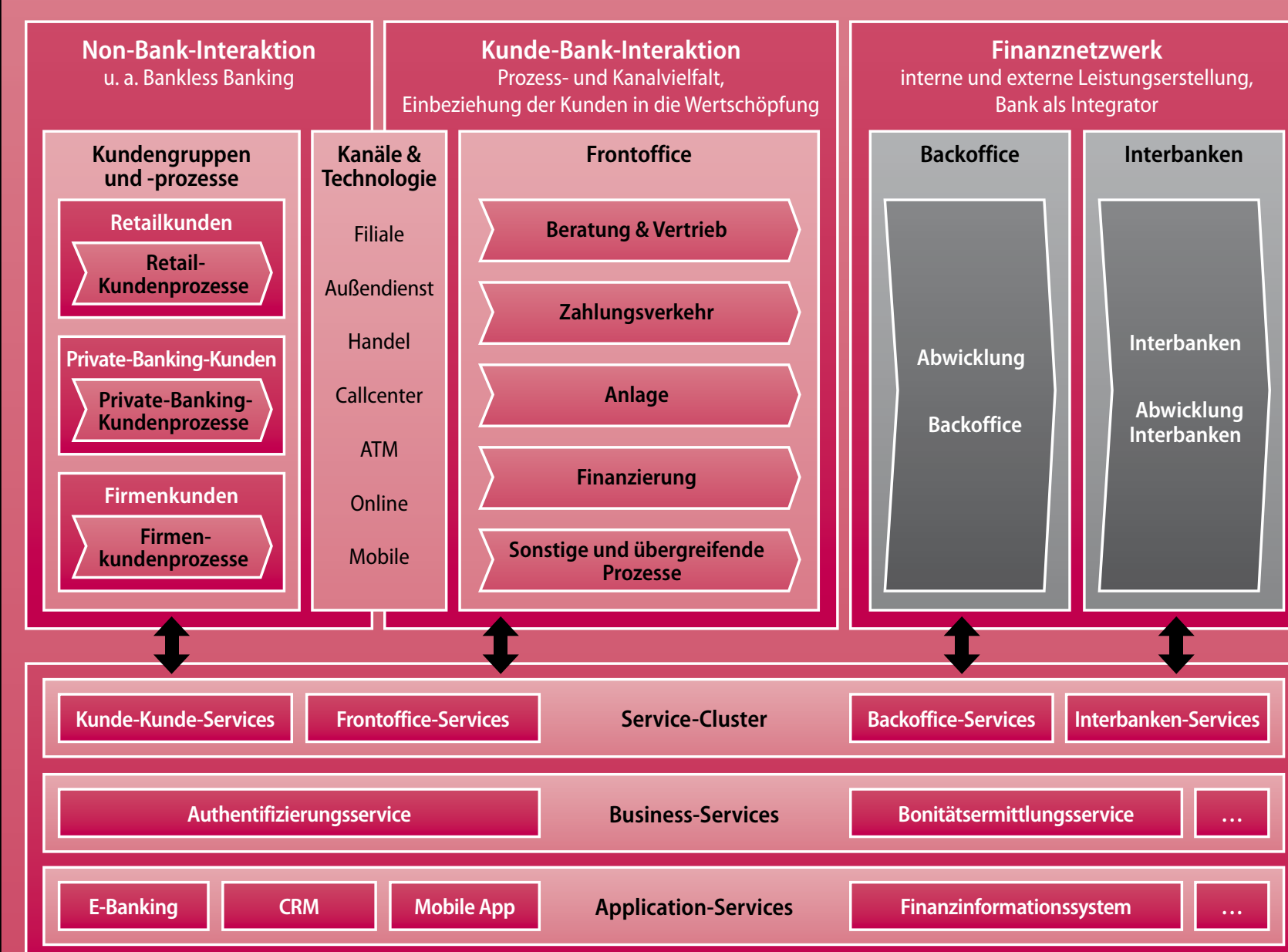

Services 


\section{Trend 1 „Interaktiver Point of Banking (POB)"}

beschreibt den Einsatz interaktiver Technologien in der stationären Beratung. Portable Geräte wie etwa Tablet-Computer, Multitouch-Tische oder sogar größere berührungsintensive Wandflächen realisieren einen interaktiven Beratungsprozess. Noch ungeklärt ist, wie weit hier auch dreidimensionale Technologien sinnvoll sind. Entsprechende 3D-Monitore sind heute auch ohne die Nutzung spezieller Brillen verfügbar und könnten auf besonders kritische Werte hinweisen oder zusätzliche Parameter in die Visualisierung aufnehmen.

\section{Trend 2 "Mobiler POB"}

baut auf der zunehmenden Intelligenz mobiler Endgeräte beim Endkunden auf und umfasst sowohl Smartphones und PCs als auch intelligente Objekte wie etwa physische Geldbörsen (z. B. Proverbial Wallet), deren Aktuatoren mit den Kontobewegungen verbunden sind und beispielsweise bei Zahlungsbewegungen Vibrationen auslösen. Von besonderer Bedeutung ist die Abstimmung mit den übrigen Interaktionskanälen zwischen Kunden und Bank.

\section{Trend 3 „,Konfigurativer POB"}

„umfasst“ die Möglichkeiten zur Entwicklung individueller Finanzprodukte. Beispielsweise lassen sich in der Filiale online oder mobil eingesetzte Konfiguratoren zur Anpassung von Laufzeiten, Produktparametern, personalisierten Aufdrucken bei Kreditkarten oder Ähnlichem verwenden. Ebenso könnten sich Endkunden Bankdienstleistungen verschiedener Anbieter aus einem Application Store herunterladen und sich dadurch ihre Bankumgebung konfigurieren.

\section{Trend 4 „Integrierter POB“}

beruht auf der Verfügbarkeit von Echtzeit-Informationen in der gesamten Banking Value Chain. Im Prinzip haben Kunden Zugriff auf die gleichen Informationsstände wie ihre Kundenberater, was Letztere vom transaktionalen Geschäft entlastet. Die mit Integration geschaffene Transparenz setzt die umfassende Konsolidierung von Informationen verschiedener Anbieter etwa mittels Business-Intelligence-Technologien voraus.

\section{Trend 5 ",Multifunktionaler POB"}

bezeichnet die Konvergenz von Geräten in der Kunde-BankInteraktion. Obgleich das dominante „Device“ noch nicht feststeht, so ist doch vorstellbar, dass Mobiltelefone auch als Kunden- und Kreditkarte oder als Coupon-Speicher im E-LoyaltyBereich Verwendung finden. Scanner am POB könnten Barcodes und zweidimensionale Codes (z. B. Microsoft Tag) dann vom Display der mobilen Geräte ablesen.

\section{Trend 6 „Öffnung des POB"}

schließlich zeigt auf Veränderungen in der Branchenstruktur. So wächst der sogenannte Nicht- beziehungsweise Parabanken-
Markt und nimmt heute bereits circa zehn Prozent des gesamten Umsatzvolumens im Bereich der Finanzdienstleistungen ein. Beispiele wie das Zahlungsinstitut oder Smava zeigen, dass neue Wettbewerber mit ihren Innovationen in den Bankenmarkt vorstoßen und Kunden für einige klassische Bankdienstleistungen bereits heute keine Banken mehr zur Abwicklung benötigen. Community-Anbieter wie Facebook und XING könnten weitere Anbieter zukünftiger Finanzdienstleistungen sein.

Vor diesem Hintergrund deutet sich an, dass die Zukunft in der Kunde-Bank-Interaktion erst begonnen hat und die großen Herausforderungen beziehungsweise Veränderungen noch bevorstehen. Neben den dargestellten technologischen Treibern unterstützen regulatorische oder bankfachliche diese Entwicklung weiter. Dabei zeigt sich, dass unter anderem aufgrund der Anforderungen an Interoperabilität und der notwendigen Investitionen die Möglichkeiten individueller Bankinstitute rasch überschritten sein könnten und zunehmende Kooperationen, beispielsweise zwischen Banken und Technologieanbietern, notwendig werden. Offen bleibt die Geschwindigkeit dieser Transformationen, da gegenwärtige Anbieter im Bankenmarkt noch von Intransparenz profitieren und daher Initiativen in Richtung verstärkter Transparenz (noch) abwartend gegenüberstehen.

\section{Literatur}

[1] Alt, R.; Bernet, B.; Eckert, C.; Etter, W.; Fischbach, M.; Kohlmann, F.; Kutsch, O.; Mansfeldt, K.; Möwes, T.; Österle, H.; Puschmann, T.; Zerndt, T. (2009). Transformation zur Bank 2015, Institut für Wirtschaftsinformatik, Universität St. Gallen.

[2] Schwabe, G.; Mogicato, R. (2009). Beratungsqualität in Banken. Was der Kunde erwartet. Was der Kunde erlebt. Institut für Informatik der Universität Zürich Solution Providers AG.

[3] Reichwald, R; Piller, F. T. (2009). Interaktive Wertschöpfung: Open Innovation, Individualisierung und neue Formen der Arbeitsteilung. Wiesbaden, Gabler.

[4] Forrester Research (Niemeyer, V.; Ensor, B.; Hesse, A.; Tincher, C.) (2009). How German Banking Customers Use Different Channels. Forrester Research.

[5] Kohlmann, F.; Alt, R. (2009). Vernetzung durch Serviceorientierung. Transformation von Banken. R. Alt, B. Bernet and T. Zerndt. Berlin, Springer: 99-128.

[6] BITKOM (09.05.2010). Fast 26 Millionen Deutsche machen Online-Banking. Bundesverband Informationswirtschaft, Telekommunikation und neue Medien e. V. Retrieved 20.05.2010 from http://www.bitkom.org/files/ documents/BITKOM_Presseinfo_Online-Banking_09_05_2010.pdf.

\section{Autoren}

Prof. Dr. Rainer Alt ist Professor für Anwendungssysteme am Institut für Wirtschaftsinformatik der Universität Leipzig und Gründer des Kompetenzzentrums Sourcing in der Finanzindustrie ( CC Sourcing).

Till Möwes ist wissenschaftlicher Mitarbeiter und Doktorand am Institut für Wirtschaftsinformatik der Universität Leipzig.

Dr. Thomas Puschmann ist Leiter des CC Sourcing am Direct Management Institute St. Gallen (DMI AG). 


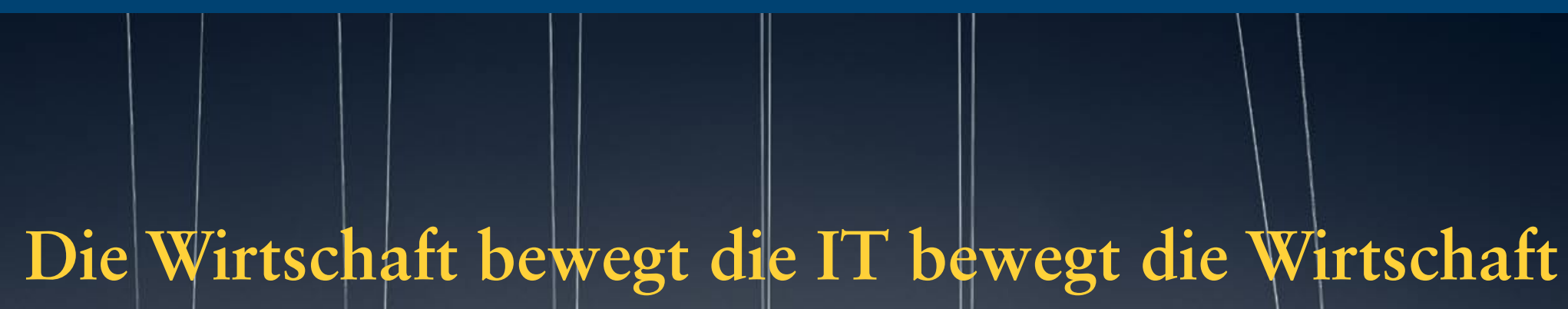
bewegt die IT bewegt die Wirtschaft ... von Wirtschaft und IT. Für Naturwissenschaftler, Mathematiker und Informatiker. Vom 7. bis 10. Oktober 2010 in Kitzbühel.

Erfahren Sie praxisnah, wie McKinsey Unternehmen, Institutionen und Individuen dabei unterstützt, komplexe Aufgabenstellungen an der Schnittstelle von Business und Technologie zu lösen. Gewinnen Sie dabei Einblicke in die vielfältigen Tätigkeitsfelder des „Business Technology Office“ und informieren Sie sich über Ihre persönlichen Karrieremöglichkeiten bei McKinsey. Bewerben Sie sich bis zum 15. August 2010 unter www.wechselwirkungen.mckinsey.de 